\title{
Uniqueness of minimal projections onto two-dimensional subspaces
}

\author{
by \\ Boris Shekhtman and LesŁaW Skrzypek (Tampa, FL)
}

\begin{abstract}
We prove that minimal projections from $L_{p}(1<p<\infty)$ onto any twodimensional subspace are unique. This result complements the theorems of W. Odyniec ([OL, Theorem I.1.3], [O3]). We also investigate the minimal number of norming points for such projections.
\end{abstract}

0. Introduction. W. Odyniec ([OL, Theorem I.1.3], [O3]) proved that minimal projections of norm greater than one from a three-dimensional Banach space onto any of its two-dimensional subspace are unique. This result can be generalized neither to the subspaces of codimension one nor to the subspaces of dimension two, unless additional assumptions on the space are considered.

However, as proved by Odyniec ([OL, Theorem I.2.22], [O1, O2]) every subspace of codimension one in $L_{p}(1<p<\infty)$ has a unique minimal projection.

In this paper we complete the picture by showing that every two-dimensional subspace of $L_{p}(1<p<\infty)$ has a unique minimal projection. Specifically we prove the following theorem:

THEOREM 0.1. Let $V$ be a two-dimensional subspace of an $L_{p}(\mu)(1<$ $p<\infty)$. Then there is a unique minimal projection from $X$ onto $V$.

We prove this theorem in Section 1. The proof depends on the number of norming points (and functionals) for minimal projections. In Section 2 we investigate one particular minimal projection and its norming pairs.

The rest of this section is devoted to general remarks and necessary definitions.

It is well known (see [IS] and [CMO]) that for every finite-dimensional subspace $V$ of a Banach space $X$ there exists a minimal projection.

2000 Mathematics Subject Classification: Primary 41A65.

Key words and phrases: approximation theory, minimal projections, uniqueness of minimal projections.

The second author was partially supported by NATO Advanced Grant. 
The problem of finding a minimal projection and related problems received the attention of many mathematicians (see [BP, CF, CL, CM1, CM2, CHM, CMO, CP, F, KTJ, R]) and it turned out to be easier in $L_{1}$ spaces than in $L_{p}$ spaces (mostly due to Theorem 1 in [CM2] which can be effectively applied in $L_{1}$ ).

The problem of uniqueness of minimal projection, however, is not well understood yet. It is clear that subspaces of $L_{1}$ usually lack uniqueness (see [CM1]) though the classical Fourier projection onto trigonometric polynomials is unique in $L_{1}$ as well as in the space of continuous functions (compare $[\mathrm{CHM}, \mathrm{FMW}])$. For necessary and sufficient conditions for the uniqueness of minimal projections onto two-dimensional subspaces of $\ell_{\infty}^{n}$ see [L3].

As far as we know, for $1<p<\infty$ there is no example of subspaces of $L_{p}$ (finite-dimensional or finite-codimensional) for which a minimal projection is not unique. Even the uniqueness of minimal projections onto trigonometric polynomials is not known.

To the best of our knowledge the only results in this direction are the previously mentioned theorem of Odyniec and the theorem of H. B. Cohen and F. E. Sullivan which states that if a minimal projection in $L_{p}(1<$ $p<\infty)$ has norm one then it is unique (see [CS]). In particular all onedimensional subspaces of $L_{p}(1<p<\infty)$ have unique minimal projection. We hope that Theorem 0.1 is a modest contribution to this list.

It is worth mentioning that the result of $\mathrm{W}$. Odyniec has been improved by G. Lewicki ([L3, Theorem 2.6.11]) by showing that a minimal projection of norm greater than one from a three-dimensional real Banach space onto any two-dimensional subspace is in fact strongly unique.

Let us introduce some basic notions, definitions and facts used in this paper. Let $S(X)$ and $B(X)$ denote the unit sphere and unit ball of a Banach space $X$.

A projection $P$ from $X$ onto a subspace $V$ is called minimal if it has the smallest possible norm, i.e.,

$$
\|P\|=\lambda(V, X)=\inf \{\|Q\|: Q \text { is a projection from } X \text { onto } V\} .
$$

The constant $\lambda(V, X)$ is called the relative projection constant.

Definition 0.2. A functional $f \in S\left(X^{*}\right)$ is a norming functional for a projection $P: X \rightarrow V$ iff $\|f \circ P\|=\|P\|$.

It is well known that if $V$ is finite-dimensional then $P$ has norming functionals (see [OL, Lemma III.2.1]).

Definition 0.3. A point $x \in S(X)$ is a norming point for a projection $P: X \rightarrow V$ iff $\|P(x)\|=\|P\|$.

If $X$ is a reflexive space and $V$ is finite-dimensional then $P$ has a norming functional $f$ and since the functional $f \circ P$ attains its norm, $P$ has a norming 
point (this is not so in general Banach spaces: the Fourier projection does not have a norming point in the space of continuous functions, see [OL, Lemma I.2.7]).

Definition 0.4. A pair $(f, x)$ is called a norming pair for a projection $P$ iff $f(P x)=\|P\|$. A set of all norming pairs for a projection $P$ is denoted by $\mathcal{E}(P)$.

As usual, for $g \in X^{*}$ and $y \in X$, the symbol $g \otimes y$ denotes the onedimensional operator from $X$ to $X$ given by $g \otimes y(x)=g(x) y$.

For the sake of completeness we state the Rudin Theorem which will be used for proving minimality of a projection given in Section 2.

Definition 0.5. Suppose that a Banach space $X$ and a topological group $G$ are related in the following manner: to every $s \in G$ corresponds a continuous linear operator $T_{s}: X \rightarrow X$ such that

$$
T_{e}=I, \quad T_{s t}=T_{s} T_{t} \quad(s \in G, t \in G) .
$$

Under these conditions, $G$ is said to act as a group of linear operators on X.

Definition 0.6. A map $L: X \rightarrow X$ commutes with $G$ if $T_{g} L T_{g}^{-1}=L$ for every $g \in G$.

Theorem 0.7 (Rudin, [W, III.B.13]). Let $X$ be a Banach space and $V$ a complemented subspace, i.e., $\mathcal{P}(X, V) \neq \emptyset$. Let $G$ be a compact group which acts as a group of linear operators on $X$ such that

(1) $T_{g}(x)$ is a continuous function of $g$ for every $x \in X$,

(2) $T_{g}(V) \subset V$ for all $g \in G$.

(3) $T_{g}$ is an isometry for all $g \in G$.

Furthermore, assume that there exists only one projection $P: X \rightarrow V$ which commutes with $G$. Then this projection is minimal.

Once we know that there is only one projection $P$ commuting with $G$ it can be easily found: fix any projection $Q$ from $X$ onto $V$; then

$$
P(x)=\int_{G} T_{g} Q T_{g^{-1}}(x) d g \quad \text { for } x \in X .
$$

This theorem, however, does not imply that this projection is the unique minimal projection as there could be projections which do not commute with $G$ but still have a minimal norm (see [S], [L1]).

\section{Proof of Theorem 0.1}

LEMMA 1.1. Let $V$ be a two-dimensional subspace of a Banach space $X$. Let $x \in S(X) \backslash V$. Then for any $\alpha>0$ there exists a projection $Q$ from $X$ onto $V$ such that $\|Q(x)\|=\alpha$. 
Proof. Let $v_{1}, v_{2} \in S(V)$ be a basis for $V$. Since $x, v_{1}, v_{2}$ are linearly independent, using the Hahn-Banach theorem we can choose

$$
f_{1} \in X^{*} \text { such that } f_{1}\left(v_{1}\right)=1 \text { and }\left.f_{1}\right|_{\operatorname{span}\left\{x, v_{2}\right\}}=0
$$

and

$$
f_{2} \in X^{*} \text { such that } f_{2}\left(v_{2}\right)=1 \text { and }\left.f_{2}\right|_{\operatorname{span}\left\{\frac{1}{\alpha} x-v_{2}, v_{1}\right\}}=0 .
$$

We have chosen $f_{1}$ and $f_{2}$ such that

$$
\begin{aligned}
& f_{1}(x)=0, \quad f_{1}\left(v_{1}\right)=1, \quad f_{1}\left(v_{2}\right)=0, \\
& f_{2}(x)=\alpha, \quad f_{2}\left(v_{1}\right)=0, \quad f_{2}\left(v_{2}\right)=1 .
\end{aligned}
$$

Now take

$$
Q=f_{1} \otimes v_{1}+f_{2} \otimes v_{2}: X \rightarrow V .
$$

From (1.1), $Q\left(v_{1}\right)=v_{1}$ and $Q\left(v_{2}\right)=v_{2}$ so $Q$ is a projection, and from (1.1),

$$
Q(x)=f_{1}(x) v_{1}+f_{2}(x) v_{2}=\alpha v_{2}
$$

hence $\|Q(x)\|=\alpha$.

THEOREM 1.2. Let $V$ be a two-dimensional subspace of a uniformly convex Banach space $X$. Let $P$ be a minimal projection from $X$ onto $V$. Then there exist at least two linearly independent norming points for $P$.

Proof. Since uniformly convex spaces are reflexive and $P$ is a compact operator and every compact operator attains its norm in a reflexive Banach space, $P$ has at least one norming point. If $\|P\|=1$ then the statement is obvious. Now, suppose that $\pm x_{0} \in S(X)$ are the only norming points for $P$. From Lemma 1.1 there is a projection $Q$ from $X$ onto $V$ such that

$$
\left\|Q\left( \pm x_{0}\right)\right\| \leq 1 / 2
$$

and by continuity we can find $\varepsilon>0$ such that

$$
\|Q(x)\|<1 \quad \text { for any } x \in B\left(x_{0}, \varepsilon\right) \cup B\left(-x_{0}, \varepsilon\right) .
$$

We now claim that there exists $\eta>0$ such that

$$
\|P(x)\|<\|P\|-\eta \quad \text { for any } x \notin B\left(x_{0}, \varepsilon\right) \cup B\left(-x_{0}, \varepsilon\right) \text { and } x \in S(X) .
$$

Indeed, otherwise for any $1 / n$ we can find $x_{n} \in S(X)$ such that $x_{n} \notin$ $B\left(x_{0}, \varepsilon\right) \cup B\left(-x_{0}, \varepsilon\right)$ and $\left\|P\left(x_{n}\right)\right\| \rightarrow\|P\|$. The sequence $\left\{P\left(x_{n}\right)\right\}$ is contained in the two-dimensional space $V$, so choosing a subsequence if necessary, we can assume that $P\left(x_{n}\right) \rightarrow y_{0}$; since $\left\|P\left(x_{n}\right)\right\| \rightarrow\|P\|$, we have $\left\|y_{0}\right\|=\|P\|$. Since uniformly convex spaces have the Banach-Saks property (see [D, Theorem III.7.1]) and the sequence $\left\{x_{n}\right\}$ is bounded in norm, we can choose a subsequence $\left\{x_{n_{k}}\right\}$ whose arithmetic means converge in norm, i.e.,

$$
y_{k}:=\frac{x_{n_{1}}+\cdots+x_{n_{k}}}{k} \rightarrow y .
$$


We will show that $y$ is a norming point for $P$ (of course $y \neq x_{0}$ and $y \neq-x_{0}$, hence a contradiction). First observe that since $\left\|x_{n}\right\|=1$, we have $\left\|y_{k}\right\| \leq 1$, which implies $\|y\| \leq 1$. Now

$$
P\left(y_{k}\right)=\frac{P\left(x_{n_{1}}\right)+\cdots+P\left(x_{n_{k}}\right)}{k} \rightarrow P(y) ;
$$

but $P\left(x_{n_{k}}\right) \rightarrow y_{0}$, hence also $P\left(y_{k}\right) \rightarrow y_{0}$. Therefore $\left\|y_{0}\right\|=\|P\|$ implies $\|P(y)\|=\|P\|$, so $y$ is a norming point for $P$ different from $\pm x_{0}$, contrary to the assumption that $\pm x_{0}$ are the only norming points for $P$.

Now for every $t \in(0,1)$ consider the projection

$$
R_{t}=t Q+(1-t) P: X \rightarrow V .
$$

If $x \in B\left(x_{0}, \varepsilon\right) \cup B\left(-x_{0}, \varepsilon\right)$ and $x \in S(X)$ then by (1.3),

$$
\begin{aligned}
\left\|R_{t}(x)\right\| & =\|t Q(x)+(1-t) P(x)\| \\
& \leq t\|Q(x)\|+(1-t)\|P(x)\| \\
& <t\|P\|+(1-t)\|P\|=\|P\| .
\end{aligned}
$$

If $x \notin B\left(x_{0}, \varepsilon\right) \cup B\left(-x_{0}, \varepsilon\right)$ and $x \in S(X)$ then by (1.4),

$$
\begin{aligned}
\left\|R_{t}(x)\right\| & =\|t Q(x)+(1-t) P(x)\| \leq t\|Q(x)\|+(1-t)\|P(x)\| \\
& <t\|Q\|+(1-t)(\|P\|-\eta) \\
& =t(\|Q\|-\|P\|+\eta)+(\|P\|-\eta),
\end{aligned}
$$

and the right hand side tends to $\|P\|-\eta$ as $t$ tends to zero. Therefore for $t_{0}$ sufficiently small, by (1.5) and (1.6),

$$
\left\|R_{t_{0}}\right\|<\|P\|
$$

which contradicts minimality of $P$.

THEOREM 1.3. Let $V$ be a two-dimensional subspace of a smooth and uniformly convex space $X$. Then there is a unique minimal projection from $X$ onto $V$.

Proof. Assume that there are two different minimal projections, say $P_{1}$ and $P_{2}$. Then $Q=\left(P_{1}+P_{2}\right) / 2$ is also a minimal projection (since $\|Q\| \leq$ $\left.\left\|\left(P_{1}+P_{2}\right) / 2\right\| \leq\left(\left\|P_{1}\right\|+\left\|P_{2}\right\|\right) / 2 \leq \lambda(V, X)\right)$. Now take any $(f, x) \in \mathcal{E}(Q)$ (see Definition 0.4) and compute

$$
\lambda(V, X)=f(Q x)=\frac{1}{2} f\left(P_{1} x\right)+\frac{1}{2} f\left(P_{2} x\right) \leq \frac{1}{2} \lambda(V, X)+\frac{1}{2} \lambda(V, X)=\lambda(V, X) ;
$$

therefore, since $f\left(P_{i} x\right) \leq\left\|P_{i}\right\|=\lambda(V, X)$,

$$
f\left(P_{1} x\right)=\lambda(V, X)=\left\|P_{1}\right\| \quad \text { and } f\left(P_{2} x\right)=\lambda(V, X)=\left\|P_{2}\right\| .
$$

As a consequence we have

$$
\mathcal{E}(Q) \subset \mathcal{E}\left(P_{1}\right), \quad \mathcal{E}(Q) \subset \mathcal{E}\left(P_{2}\right),
$$

i.e., any norming pair for $Q$ is also a norming pair for $P_{1}$ and $P_{2}$. 
Since $Q$ is a minimal projection, by Theorem 1.2, there are two linearly independent norming points $x_{1}$ and $x_{2}$ for $Q$. Let $\left(f_{1}, x_{1}\right)$ and $\left(f_{2}, x_{2}\right)$ be corresponding norming pairs for $Q$. Observe that

$$
\left.f_{1}\right|_{V^{*}},\left.f_{2}\right|_{V^{*}} \text { are linearly independent. }
$$

Indeed, if not then $f_{1}= \pm f_{2}$ and $f_{1}\left(Q x_{1}\right)=f_{1}\left(Q\left( \pm x_{2}\right)\right)=\|Q\|$. Hence

$$
f_{1}\left(Q\left(\frac{x_{1}+\left( \pm x_{2}\right)}{2}\right)\right)=\|Q\|
$$

so $\left\|\left(x_{1}+\left( \pm x_{2}\right)\right) / 2\right\|=1$, which is not possible if $X$ is strictly convex.

From (1.7),

$$
f_{i}\left(P_{1} x_{i}\right)=\left\|P_{1}\right\|, \quad f_{i}\left(P_{2} x_{i}\right)=\left\|P_{2}\right\| .
$$

Therefore

$$
\left(P_{1}^{*} f_{i}\right)\left(x_{i}\right)=\left\|P_{1}\right\|=\lambda(V, X), \quad\left(P_{2}^{*} f_{i}\right)\left(x_{i}\right)=\left\|P_{2}\right\|=\lambda(V, X) .
$$

It now follows that $\left(P_{1}^{*} f_{i}\right) /\left\|P_{1}\right\|$ and $\left(P_{2}^{*} f_{i}\right) /\left\|P_{2}\right\|$ are two norming functionals for $x_{i}$. Since $X$ is smooth they have to be equal. Hence

$$
P_{1}^{*} f_{i}=P_{2}^{*} f_{i}
$$

and since the $\left.f_{i}\right|_{V^{*}} \operatorname{span} V^{*}((1.8))$ we have $P_{1}^{*}=P_{2}^{*}$. Hence $P_{1}=P_{2}$.

Corollary 1.4. Let $V$ be a two-dimensional subspace of $L_{p}(\mu)$ with $1<p<\infty$. Then there is a unique minimal projection from $L_{p}(\mu)$ onto $V$ (this covers both classical cases $L_{p}[0,1]$ and $\ell_{p}$ ).

2. Norming pairs. It was seen in the previous section that there are at least two linearly independent norming points for a minimal projection onto a two-dimensional subspace. In this section we show that there are at least six norming points, altogether, for such a projection. We show, by means of an example, that the number six cannot be increased.

Theorem 2.1. A minimal projection from $L_{p}(\mu)($ with $1<p<\infty)$ onto a two-dimensional subspace has at least six different norming functionals $\pm f_{1}, \pm f_{2}, \pm f_{3}$. Moreover the set of restrictions to $V^{*}$ of these functionals, $\pm\left. f_{1}\right|_{V^{*}}, \pm\left. f_{2}\right|_{V^{*}}, \pm\left. f_{3}\right|_{V^{*}}$, contains six different elements.

Proof. By [OL, Theorem III.2.8 and Remark III.2.9], every set $C$ such that

$$
C \cup-C=\left\{\text { the set of norming functionals of } P \text { restricted to } V^{*}\right\}
$$

and

$$
C \cap-C=\emptyset
$$

is linearly dependent over $V^{*}$. But by Theorem 1.2 and reasoning as in the proof of Theorem 1.3 (see (1.8)) we have at least two norming functionals 
for $P$ which are linearly independent over $V^{*}$. Hence $C$ has to contain at least three elements.

THEOREM 2.2. A minimal projection from $L_{p}(\mu)$ (with $\left.1<p<\infty\right)$ onto a two-dimensional subspace has at least six different norming points $\pm x_{1}, \pm x_{2}, \pm x_{3}$.

Proof. By the previous theorem there are three norming functionals for $P$ such that

$$
\left.f_{1}\right|_{V^{*}},\left.f_{2}\right|_{V^{*}},\left.f_{3}\right|_{V^{*}} \text { are three different functionals. }
$$

To these functionals correspond three norming points $x_{1}, x_{2}, x_{3}$. Let

$$
g_{i}=\frac{f_{i} \circ P}{\|P\|} .
$$

By (2.1), $g_{1}, g_{2}, g_{3}$ are three different functionals on $X^{*}$ of norm one. Also

$$
g_{i}\left(x_{i}\right)=1 \text {. }
$$

Now if $x_{i}=x_{j}$ (for some $i \neq j \in\{1,2,3\}$ ) then $g_{i}$ and $g_{j}$ are norming functionals for the same point $x=x_{i}=x_{j}$. Since the $L_{p}(\mu)$ is smooth, that would imply $g_{i}=g_{j}$, a contradiction. Hence $x_{1}, x_{2}, x_{3}$ are all diferent.

TheOREM 2.3. Let $P$ be a minimal projection from $\ell_{p}^{3}$ onto a two-dimensional subspace $V$. Let $W=\left\{x \in \ell_{p}^{n}:\left(x_{1}, x_{2}, x_{3}\right) \in V\right.$ and $x_{4}=\cdots=$ $\left.x_{n}=0\right\}$. Take a projection $Q$ from $\ell_{p}^{n}$ onto $W$ defined by

$$
Q\left(x_{1}, x_{2}, x_{3}, x_{4}, \ldots, x_{n}\right)=\left(P\left(x_{1}, x_{2}, x_{3}\right), 0, \ldots, 0\right) .
$$

Then $Q$ is also a minimal projection having the same number of norming points and norming functionals as $P$.

Proof. By the very construction of $Q$, if $x=\left(x_{1}, \ldots, x_{n}\right)$ is a norming point for $Q$ then $x_{4}=\cdots=x_{n}=0$. If $f=\left(f_{1}, \ldots, f_{n}\right)$ is a norming functional for $Q$ then by the form of norming functionals (i.e., $f_{i}=\operatorname{sgn}\left(a_{i}\right)$. $\left|a_{i}\right|^{p / q}$ ) and the form of $Q$ we get $f_{4}=\cdots=f_{n}=0$. Hence $\|Q\|=\|P\|$, and moreover

$$
\begin{gathered}
x=\left(x_{1}, \ldots, x_{n}\right) \text { is a norming point for } Q \\
x=\left(x_{1}, x_{2}, x_{3}\right) \text { is a norming point for } P
\end{gathered}
$$

and

$$
\begin{aligned}
& f=\left(f_{1}, \ldots, f_{n}\right) \text { is a norming functional for } Q \\
& f=\left(f_{1}, f_{2}, f_{3}\right) \text { is a norming functional for } P .
\end{aligned}
$$

Since $L: \ell_{p}^{n} \rightarrow \ell_{p}^{3}$ given by $L\left(x_{1}, \ldots, x_{n}\right)=\left(x_{1}, x_{2}, x_{3}, 0, \ldots, 0\right)$ is a norm one projection, by [OL, Proposition I.3.1] the projection $Q$ is also minimal. 
Now we will compute the norm, all norming points and all norming functionals for a particular minimal projection.

THEOREM 2.4. Let $f=(1,1,1) \in \ell_{q}^{3}$ be a representation of a functional. Then $P: \ell_{p}^{3} \rightarrow \operatorname{ker} f$ given by

$$
P=\operatorname{Id}-\frac{1}{3}(1,1,1) \otimes(1,1,1)
$$

is a minimal projection for any $1 \leq p \leq \infty$.

Proof. First we will prove that $P$ given by (2.2) is indeed a minimal projection. We will use the Rudin Theorem. Observe that the operators

$$
L_{\sigma}\left(x_{1}, x_{2}, x_{3}\right):=\left(x_{\sigma(1)}, x_{\sigma(2)}, x_{\sigma(3)}\right)
$$

(where $\sigma$ is any permutation of $\{1,2,3\}$ ) are isometries in $\ell_{p}^{3}$. Furthermore

$$
L_{\sigma}(\operatorname{ker}(1,1,1)) \subset \operatorname{ker}(1,1,1) .
$$

Now according to Theorem 0.7 it is enough to prove that $P$ is the only projection which commutes with $L_{\sigma}$.

Any projection $Q: \ell_{p}^{3} \rightarrow \operatorname{ker}(1,1,1)$ is given by

$$
Q x=x-(1,1,1) \otimes\left(v_{1}, v_{2}, v_{3}\right), \quad \text { where } \quad v_{1}+v_{2}+v_{3}=1 .
$$

If $Q$ commutes with $L_{\sigma}$, then

$$
\left((1,1,1) \otimes\left(v_{1}, v_{2}, v_{3}\right)\right) \circ L_{\sigma}=L_{\sigma}\left((1,1,1) \otimes\left(v_{1}, v_{2}, v_{3}\right)\right) .
$$

Taking the value at $x=\left(x_{1}, x_{2}, x_{3}\right)$ on both sides of the above equality results in

$$
\begin{aligned}
\left(\sum_{i=1}^{3} x_{i}, \sum_{i=1}^{3} x_{i}, \sum_{i=1}^{3} x_{i}\right) \cdot & \left(v_{1}, v_{2}, v_{3}\right) \\
& =\left(\sum_{i=1}^{3} x_{i}, \sum_{i=1}^{3} x_{i}, \sum_{i=1}^{3} x_{i}\right) \cdot\left(v_{\sigma(1)}, v_{\sigma(2)}, v_{\sigma(3)}\right),
\end{aligned}
$$

for any $\sigma \in S_{3}$ and any $x=\left(x_{1}, x_{2}, x_{3}\right)$. Therefore $v_{1}=v_{2}=v_{3}$ and since $v_{1}+v_{2}+v_{3}=1$ we have

$$
v_{1}=v_{2}=v_{3}=1 / 3 .
$$

Hence $Q=P$. On the other hand, it is easy to see that $P$ indeed commutes with $L_{\sigma}$. Therefore $P$ is minimal.

Now we will restrict ourselves to $p=4$.

Theorem 2.5. Let $p=4$. Then the minimal projection from Theorem 2.4 (see (2.2)) has exactly six norming points 


$$
\begin{array}{ll}
x_{0}=\frac{1}{\left(2+2^{4 / 3}\right)^{1 / 4}}\left(2^{1 / 3},-1,-1\right), & x_{3}=-x_{0}, \\
x_{1}=\frac{1}{\left(2+2^{4 / 3}\right)^{1 / 4}}\left(-1,2^{1 / 3},-1\right), & x_{4}=-x_{1}, \\
x_{2}=\frac{1}{\left(2+2^{4 / 3}\right)^{1 / 4}}\left(-1,-1,2^{1 / 3}\right), & x_{5}=-x_{2},
\end{array}
$$

and exactly six norming functionals

$$
\begin{array}{ll}
f_{0}=\frac{1}{\left(2+2^{4}\right)^{3 / 4}}\left(2^{3},-1,-1\right), & f_{3}=-f_{0}, \\
f_{1}=\frac{1}{\left(2+2^{4}\right)^{3 / 4}}\left(-1,2^{3},-1\right), & f_{4}=-f_{1}, \\
f_{2}=\frac{1}{\left(2+2^{4}\right)^{3 / 4}}\left(-1,-1,2^{3}\right), & f_{5}=-f_{3} .
\end{array}
$$

Moreover,

$$
\|P\|=\lambda\left(\operatorname{ker}(1,1,1), \ell_{p}^{3}\right)=\frac{1}{3}\left(1+2^{3}\right)^{1 / 4}\left(1+2^{1 / 3}\right)^{3 / 4} .
$$

Proof. The projection $P$ from (2.2) is given by

$$
P\left(x_{1}, x_{2}, x_{3}\right)=\frac{1}{3}\left(2 x_{1}-x_{2}-x_{3},-x_{1}+2 x_{2}-x_{3},-x_{1}-x_{2}+2 x_{3}\right),
$$

therefore the problem of finding its norm and all norming points is equivalent to finding the maximum of the function

$$
\begin{aligned}
& h\left(x_{1}, x_{2}, x_{3}\right) \\
& =\left(\frac{2 x_{1}-x_{2}-x_{3}}{3}\right)^{4}+\left(\frac{-x_{1}+2 x_{2}-x_{3}}{3}\right)^{4}+\left(\frac{-x_{1}-x_{2}+2 x_{3}}{3}\right)^{4}
\end{aligned}
$$

in the set $x_{1}^{4}+x_{2}^{4}+x_{3}^{4}=1$, and finding all points at which this maximum is attained.

Let

$$
\begin{array}{ll}
z_{1}=\frac{2 x_{1}-x_{2}-x_{3}}{3}, & z_{2}=\frac{-x_{1}+2 x_{2}-x_{3}}{3}, \\
z_{3}=\frac{-x_{1}-x_{2}+2 x_{3}}{3}, & d=\frac{x_{1}+x_{2}+x_{3}}{3} .
\end{array}
$$

Then the above problem is equivalent to finding the maximum and all points at which this maximum is attained for the following function:

$$
f\left(z_{1}, z_{2}, z_{3}, d\right)=z_{1}^{4}+z_{2}^{4}+z_{3}^{4}
$$

in the set

$$
\left(z_{1}+d\right)^{4}+\left(z_{2}+d\right)^{4}+\left(z_{3}+d\right)^{4}=1 \quad \text { and } \quad z_{1}+z_{2}+z_{3}=0
$$


Using the standard Lagrange multiplier method we construct the function

$$
\begin{aligned}
\varphi\left(z_{1}, z_{2}, z_{3}, d\right)= & z_{1}^{4}+z_{2}^{4}+z_{3}^{4} \\
& -\lambda_{1}\left(\left(z_{1}+d\right)^{4}+\left(z_{2}+d\right)^{4}+\left(z_{3}+d\right)^{4}\right) \\
& -\lambda_{2}\left(z_{1}+z_{2}+z_{3}\right)
\end{aligned}
$$

and in particular we find that $z_{1}, z_{2}, z_{3}$ have to satisfy the equations

$$
g\left(z_{1}\right)=g\left(z_{2}\right)=g\left(z_{3}\right)=0, \text { where } g(x)=4 x^{3}-4 \lambda_{1}(x+d)^{3}-\lambda_{2} .
$$

Now assume that $z_{1}, z_{2}, z_{3}$ are distinct. Then by $(2.12), z_{1}, z_{2}, z_{3}$ will be three distinct zeros of $g$. That implies $\lambda_{1} \neq 0$ (in that case $g$ has only one zero), $\lambda_{1} \neq 1$ (in that case $g$ is a polynomial of degree 2 , hence has at most two zeros) and

$$
g(x)=\left(4-4 \lambda_{1}\right)\left(x-z_{1}\right)\left(x-z_{2}\right)\left(x-z_{3}\right) .
$$

Now comparing the coefficients of $g$ in (2.12) and (2.13) gives

$$
z_{1}+z_{2}+z_{3}=\frac{3 \lambda_{1} d}{1-\lambda_{1}} .
$$

On the other hand, $z_{1}+z_{2}+z_{3}=0$, hence $d=0$. But clearly a fourtuple $\left(z_{1}, z_{2}, z_{3}, 0\right)$ is not a maximum point of the function (2.11) since $f\left(z_{1}, z_{2}, z_{3}, 0\right)=1$. Thus we proved that

$$
z_{1}=z_{2} \quad \text { or } \quad z_{2}=z_{3} \quad \text { or } \quad z_{3}=z_{1} \text {, }
$$

which is equivalent to

$$
x_{1}=x_{2} \quad \text { or } \quad x_{2}=x_{3} \quad \text { or } \quad x_{3}=x_{1} .
$$

By symmetry it is enough to let $x_{2}=x_{3}$. Letting $x_{2}=x_{3}$ in (2.9) we have to find the maximum (and all points at which this maximum is attained) of the function

$$
h\left(x_{1}, x_{2}\right)=\frac{2+2^{4}}{3^{4}}\left(x_{1}-x_{2}\right)^{4} \quad \text { in the set } \quad x_{1}^{4}+2 x_{2}^{4}=1 .
$$

This can be easily solved by using Lagrange multipliers and together with (2.14) it leads to (2.6). Note that (2.7) follows immediately from (2.6).

Using Theorems 2.3 and 2.5 we obtain

Corollary 2.6. For any $\ell_{4}^{n}$ we can construct a two-dimensional subspace $V$ of $\ell_{4}^{n}$ such that the minimal projection $P$ from $\ell_{4}^{n}$ onto $V$ has only six norming functionals and six norming points.

REMARK 2.7. Theorem 2.2 is not true if the word "minimal" is dropped: we can easily find a projection (not minimal of course) which has only two different norming points. For instance,

$$
Q=\operatorname{Id}-(1,1,1) \otimes(0,0,1)
$$

has only two norming points $\pm\left(1 / 2^{1 / p}, 1 / 2^{1 / p}, 0\right)$. 


\section{References}

[BP] M. Baronti and P. Papini, Norm one projections onto subspaces of $\ell_{p}$, Ann. Mat. Pura Appl. 152 (1988) 53-61.

[CL] B. L. Chalmers and G. Lewicki, Symmetric spaces with maximal projection constants, J. Funct. Anal. 200 (2003), 1-22.

[CM1] B. L. Chalmers and F. T. Metcalf, The determination of minimal projections and extensions in $L^{1}$, Trans. Amer. Math. Soc. 329 (1992), 289-305.

[CM2] -, - A characterization and equations for minimal projections and extensions, J. Operator Theory 32 (1994), 31-46.

[CF] E. W. Cheney and C. Franchetti, Minimal projections in $L_{1}$-space, Duke Math. J. 43 (1976), 501-510.

[CHM] E. W. Cheney, C. R. Hobby, P. D. Morris, F. Schurer and D. E. Wulbert, On the minimal property of the Fourier projection, Trans. Amer. Math. Soc. 143 (1969), 249-258.

[CMO] E. W. Cheney and P. D. Morris, On the existence and characterization of minimal projections, J. Reine Angew. Math. 270 (1974), 61-76.

[CP] E. W. Cheney and K. H. Price, Minimal projections, in: Approximation Theory (Lancaster, 1969), A. Talbot (ed.), Academic Press, London, 1970, 261-289.

[CS] H. B. Cohen and F. E. Sullivan, Projecting onto cycles in smooth, reflexive Banach spaces, Pacific J. Math. 34 (1970), 355-364.

[D] J. Diestel, Geometry of Banach Spaces, Lecture Notes in Math. 485, Springer, Berlin, 1975.

[FMW] S. D. Fisher, P. D. Morris and D. E. Wulbert, Unique minimality of Fourier projections, Trans. Amer. Math. Soc. 265 (1981), 235-246.

[F] C. Franchetti, Projections onto hyperplanes in Banach spaces, J. Approx. Theory 38 (1983), 319-333.

[IS] J. R. Isbell and Z. Semadeni, Projection constants and spaces of continuous functions, Trans. Amer. Math. Soc. 107 (1963), 38-48.

[KTJ] H. König and N. Tomczak-Jaegermann, Norms of minimal projections, J. Funct. Anal. 119 (1994), 253-280.

[L1] G. Lewicki, On the unique minimality of the Fourier-type extensions in $L_{1}$-space, in: Function Spaces (Poznań, 1998), H. Hudzik and L. Skrzypczak (eds.), Lecture Notes Pure Appl. Math. 213, Dekker, New York, 2000, 337-345.

[L2] - Strong unicity criterion in some space of operators, Comment. Math. Univ. Carolin. 34 (1993), 81-87.

[L3] - Best approximation in spaces of bounded linear operators, Dissertationes Math. 330 (1994).

[O1] V. P. Odinec, On uniqueness of minimal projections in Banach space, Dokl. Akad. Nauk SSSR 220 (1975), 779-781 (in Russian).

[O2] - Conditions for uniqueness of minimal projections with unit norm, Mat. Zametki 22 (1977), no. 6, 45-49 (in Russian).

[O3] -, The uniqueness of minimal projection, Soviet Math. (Iz. VUZ) 22 (1978), no. $2,64-66$.

[OL] W. Odyniec and G. Lewicki, Minimal Projections in Banach Spaces, Lecture Notes in Math. 1449, Springer, Berlin, 1990.

[R] S. Rolewicz, On projections on subspaces of codimension one, Studia Math. 44 (1990), 17-19. 
[S] L. Skrzypek, The uniqueness of minimal projections in smooth matrix spaces, J. Approx. Theory 107 (2000), 315-336.

[W] P. Wojtaszczyk, Banach Spaces for Analysts, Cambridge Univ. Press, 1991.

Department of Mathematics

University of South Florida

4202 E. Fowler Ave., PHY 114

Tampa, FL 33620-5700, U.S.A.

E-mail: boris@math.usf.edu

skrzypek@chuma.cas.usf.edu

Received March 9, 2004

Revised version January 20, 2005 